\title{
Cerebral hemodynamics of the aging brain: risk of Alzheimer disease and benefit of aerobic exercise
}

\author{
Takashi Tarumi ${ }^{1,2}$ and Rong Zhang ${ }^{1,2,3 *}$ \\ ${ }^{1}$ Institute for Exercise and Environmental Medicine, Texas Health Presbyterian Hospital Dallas, Dallas, TX, USA \\ ${ }^{2}$ Department of Internal Medicine, University of Texas Southwestern Medical Center, Dallas, TX, USA \\ ${ }^{3}$ Department of Neurology and Neurotherapeutics, Alzheimer's Disease Center, University of Texas Southwestern Medical Center, Dallas, TX, USA
}

Edited by:

Patrice Brassard, Laval University,

Canada

Reviewed by:

Andrea Weinstein, University of

Pittsburgh, USA

Jean Chen, University of Toronto,

Canada

\section{${ }^{*}$ Correspondence:}

Rong Zhang, Institute for Exercise and Environmental Medicine, Texas Health Presbyterian Hospital Dallas, University of Texas Southwestern Medical Center at Dallas, 7232

Greenville Ave, Dallas, TX 75231,

USA

e-mail: rongzhang@texashealth.org
Alzheimer disease (AD) and cerebrovascular disease often coexist with advanced age. Mounting evidence indicates that the presence of vascular disease and its risk factors increase the risk of $A D$, suggesting a potential overlap of the underlying pathophysiological mechanisms. In particular, atherosclerosis, endothelial dysfunction, and stiffening of central elastic arteries have been shown to associate with AD. Currently, there are no effective treatments for the cure and prevention of AD. Vascular risk factors are modifiable via either pharmacological or lifestyle intervention. In this regard, habitual aerobic exercise is increasingly recognized for its benefits on brain structure and cognitive function. Considering the well-established benefits of regular aerobic exercise on vascular health, exercise-related improvements in brain structure and cognitive function may be mediated by vascular adaptations. In this review, we will present the current evidence for the physiological mechanisms by which vascular health alters the structural and functional integrity of the aging brain and how improvements in vascular health, via regular aerobic exercise, potentially benefits cognitive function.

Keywords: cerebral hemodynamics, aging, Alzheimer's disease, vascular dementia, regular aerobic exercise

\section{INTRODUCTION}

Alzheimer's disease (AD) is a devastating neurological disorder characterized by progressive deterioration of brain structure and function (Querfurth and LaFerla, 2010). Advanced age is the strongest risk factor for $\mathrm{AD}$ such that the risk doubles every 5 years after age of 65 . Due to the rapid aging of the population, the prevalence of $\mathrm{AD}$ is facing an exponential growth. However, there remains a dearth of effective treatments, cures, and most importantly preventions (Thies and Bleiler, 2013).

Mounting evidence indicates that vascular disease and risk factors not only elevate the risk of vascular dementia $(\mathrm{VaD})$ but also AD (de la Torre, 2004). Traditional views on the etiology of AD and $\mathrm{VaD}$ have been divergent. $\mathrm{AD}$ develops as a result of brain amyloid depositions, leading to a pathological cascade of neurodegeneration and cognitive impairment (Selkoe, 1991; Hardy and Selkoe, 2002). On contrary, VaD is attributed to cerebral hypoperfusion and ischemia that are associated with impairment of synaptic activity and protein synthesis, glutamate excitotoxicity, and neuronal apoptosis (Hossmann, 1994; Gorelick et al., 2011). Despite these traditional perspectives, large populationbased prospective studies have demonstrated that vascular risk factors in midlife, such as systolic hypertension and hyperlipidemia, led to a significant elevation of $\mathrm{AD}$ risk in later life (Kivipelto et al., 2001).

Vascular risk and dysfunction are modifiable through lifestyle modifications (Ornish et al., 1990). In particular, physical activity has been shown to improve cognitive outcomes in patients with mild cognition impairment (Lautenschlager et al., 2008). In contrast to the pharmacological interventions, habitual physical activity such as "regular aerobic exercise" is low cost, has virtually no adverse effects, and can be important for primary prevention of AD (Selkoe, 2012). Nonetheless, the physiological benefits of regular aerobic exercise on $\mathrm{AD}$ prevention are not completely understood and are only supported by a limited amount of data. Accordingly, the primary objective of this review is to overview the current evidence of the association between vascular aging and $\mathrm{AD}$ pathology. Secondarily, we will extend our discussion to the potential mechanism by which regular aerobic exercise may attenuate $\mathrm{AD}$ pathology via improvements in vascular health.

\section{CEREBROVASCULAR ANATOMY AND BLOOD FLOW REGULATION}

The brain relies critically on a constant supply of blood due to its high rate of oxidative metabolism and lack of energy substrate. $\sim 15 \%$ of cardiac output is directed to the brain which only weighs $\sim 2 \%$ of body mass yet accounts for $\sim 20 \%$ of total blood glucose and oxygen utilizations (Attwell et al., 2010). To sustain the high volume blood supply, cerebrovascular resistance is low. However, this makes the brain sensitive to changes in cerebral blood flow (CBF) during hypo- or hypertension (Faraci and Heistad, 1990). Importantly, the majority of cerebrovascular resistance is controlled outside of the parenchyma by extracerebral arteries (i.e., large cerebral arteries and pial arterioles) while intracerebral arterioles and capillaries account for the remaining resistance (Faraci and Heistad, 1990).Therefore, the coordinated adjustment of extra- and intracerebral arteries in response to changes in perfusion pressure is crucial in maintaining adequate perfusion and normal brain function. 
Cerebral autoregulation (CA) maintains CBF relatively constant in the face of changes in arterial pressure (Lassen, 1959; Paulson et al., 1990). In particular, extracerebral adjustment of arteriolar resistance ensures adequate perfusion to the parenchyma (Rowbotham and Little, 1965; Faraci and Heistad, 1990). Watershed areas of deep and periventricular white matter are particularly vulnerable to hypoperfusion and hypoxia, especially with age-related vascular disease and dysfunction (Rowbotham and Little, 1965; Faraci and Heistad, 1990; Matsushita et al., 1994). Dysregulation of CBF and perfusion pressure may cause ischemic brain injuries such as white matter hyperintensities (WMH) and silent infarcts which are often associated AD pathology (Snowdon et al., 1997). Below we will discuss steady-state and dynamic regulations of CBF and its implications on the aging brain and $\mathrm{AD}$ pathology.

\section{REGULATION OF STEADY-STATE CBF}

CBF decreases with advancing age and is lower in patients with $\mathrm{AD}$ compared with age-matched healthy individuals (Leenders et al., 1990; Matsuda, 2001). Age- and/or AD-related reductions in $\mathrm{CBF}$ are, at least in part, explained by the concurrent atrophy or lower metabolic rate of brain tissue (Matsuda, 2001). However, the recent evidence demonstrating the association between vascular disease and a higher incidence of $\mathrm{AD}$ also raises a potential hypothesis that dysregulation of CBF may cause an insufficient supply of energy and nutrients and accelerate the AD pathology (de la Torre, 2004). In healthy individuals, CBF and metabolism are tightly coupled via the neurovascular unit which regulates microvascular resistance upon neuronal activities (Attwell et al., 2010). In contrast, in patients with amnestic mild cognitive impairment, a prodromal stage of $\mathrm{AD}$, global $\mathrm{CBF}$ and metabolic rate of oxygen were reduced and cerebrovascular resistance was elevated when compared with the normal controls (Liu et al., 2013). Furthermore, a linear relation that was observed in healthy subjects between global CBF and metabolic rate of oxygen was absent in patients with amnestic mild cognitive impairment. These findings suggest the presence of neurovascular decoupling of CBF and metabolism in the individual who is at greater risk of AD. Pathologically, cerebral hypoperfusion or hypoxic ischemia have been shown to increase amyloid plaque depositions, the pathological hallmark of $\mathrm{AD}$, which precedes neurodegeneration and cognitive impairment (Zhang et al., 2007; Okamoto et al., 2012).

Physiological mechanisms by which vascular disease and risk factors promote the $\mathrm{AD}$ pathology are likely to be multi-factorial and related to a number of changes in vascular structure and function (Breteler, 2000). Although the relative degree to which each component of vascular abnormalities plays a role remains unclear, endothelial dysfunction, a hallmark of vascular aging, has been observed in patients with AD (Dede et al., 2007). Vascular endothelium, the most internal layer of the arterial wall, is susceptible to blood-derived chemical and mechanical stimuli. In response to these stimuli, the endothelium releases vasoactive substances to maintain vascular homeostasis. In particular, constitutive nitric oxide (NO) plays a crucial role in maintaining vasomotor tone and its dynamic regulation facilitates functional hyperemia in response to changes in metabolic demand (Fujii et al., 1991; Dietrich et al., 1996). Importantly, NO also inhibits atherogenesis by reducing oxidative modification of low-density lipoprotein (LDL) cholesterol and preventing the proliferation of vascular smooth muscle cells (Davignon and Ganz, 2004). Oxidation of LDL has been proposed as a major mechanism of the atherosclerotic process (Davignon and Ganz, 2004). In patients with $\mathrm{AD}$, age-related impairment of endothelial function is exacerbated such that endothelium-dependent NO-mediated vasodilatory function, as assessed by brachial flow-mediated dilation, is lower when compared with healthy subjects (Dede et al., 2007). Consistently, exposure of cerebrovascular endothelium to amyloid- $\beta(A \beta)$ peptide acutely impairs endothelium-dependent vasodilation by augmenting oxidative stress (Thomas et al., 1996). Moreover, autopsy studies have demonstrated a greater atherosclerotic burden in patients with $\mathrm{AD}$ than healthy controls (Roher et al., 2003). Collectively, these findings suggest that cerebral endothelial dysfunction, $A \beta$ induced vasoconstriction, and atherosclerotic encroachment of cerebral arteries may elevate cerebrovascular resistance leading to brain hypoperfusion which in turn may accelerate the AD pathology.

$\mathrm{AD}$ and atherosclerosis also share the common genetic risk factors, such as the $\varepsilon 4$ allele of apolipoprotein E (APOE4) (Casserly and Topol, 2004). Apolipoprotein E is a polymorphic protein arising from three alleles (i.e., $\varepsilon 2, \varepsilon 3$, and $\varepsilon 4$ ), which differs by only a single amino acid substitute. Yet these changes have the profound influences on the brain and peripheral lipid metabolism (Mahley and Rall, 2000). Although the underlying cellular and molecular mechanism remains to be established, APOE4 is associated with greater $\mathrm{A} \beta$ depositions in the brain ( $\mathrm{Bu}, 2009$; Kim et al., 2009) and higher plasma concentrations of LDL. Increased LDL elevates the risk of atherogenesis, especially when associated with endothelial dysfunction (Wilson et al., 1994, 1996).

\section{REGULATION OF DYNAMIC CBF: BEAT-TO-BEAT LOW FREQUENCY OSCILLATIONS}

The brain is susceptible to dynamic changes in arterial pressure which spontaneously oscillates at the low frequencies below heart rate at rest (Zhang et al., 2000). CA during dynamic beat-to-beat changes in arterial pressure more or less is a frequency dependent phenomenon which may dampen oscillations of CBF in response to changes in arterial pressure at the low frequencies (Lassen, 1959; Paulson et al., 1990; Zhang et al., 1998). CA is an inherent property of cerebral arteries and arterioles that are controlled by myogenic, neurogenic, and metabolic mechanisms. In effect, CA ensures a constant supply of oxygen and nutrients during hypotension while attenuating hyperperfusion during hypertension.

Contrary to the general pattern of age-related impairment of vascular function, CA appears to remain intact in older adults (van Beek et al., 2008). Furthermore, recent studies suggest that CA is also preserved in patients with AD (Claassen et al., 2009; Zazulia et al., 2010), although the presence of cerebral amyloid angiopathy, which often accompanies advanced AD pathology, may alter CA. Cerebral amyloid angiopathy is characterized by the accumulation of $A \beta_{1-40}$ proteins in the cerebral vessel wall (Christie et al., 2001). A $\beta_{1-40}$ is highly toxic to cerebral vasculature such that that exogenous application of $A \beta_{1-40}$ impairs 
endothelial-dependent vasodilation and exaggerates vasoconstriction (Thomas et al., 1996). Using a transgenic mouse model of $\mathrm{AD}$, profound impairment of CA has been observed (Niwa et al., 2002). Of note, the inconsistent findings between the preclinical and clinical studies of $\mathrm{CA}$ in $\mathrm{AD}$ clearly warrants future studies to determine whether CA is indeed impaired in $\mathrm{AD}$ and whether changes in CA are related to the $\mathrm{AD}$ onset or progression.

$\mathrm{AD}$ may also impair cerebral tissue oxygenation during dynamic changes in CBF. Cerebral tissue oxygenation, measured non-invasively be near-infrared spectroscopy, predominantly reflects the oxygen saturation of venous blood (Madsen and Secher, 1999; Claassen et al., 2006; Rowley et al., 2007; Murkin and Arango, 2009). In patients with AD, transfer function gain of cerebral tissue oxygenation in response to changes in $\mathrm{CBF}$ has been reported lower while the phase was reduced when compared with controls subjects (van Beek et al., 2010). These findings suggest that more arterial oxygen is transmitted to the venous circulation and a possibility of microvascular dysfunction in which the brain tissue is less able to extract oxygen from the arterial blood. If this is the case, it can be speculated that neurovascular coupling of CBF and metabolism may be disrupted during hemodynamic challenges in patients with $\mathrm{AD}$.

\section{REGULATION OF DYNAMIC CBF: CARDIAC FREOUENCY}

The human heart is an intermittent pump which generates a stroke volume every cardiac cycle. During healthy youth, left ventricular afterload, as well as systolic blood pressure generated to overcome the afterload, is low (Avolio et al., 1983). In addition, the compliance of central arteries effectively dampens the hemodynamic pulsations via the Windkessel effect and creates a continuous blood flow in the microcirculation of peripheral vascular beds (Nichols et al., 2005). With advancing age, central elastic arteries stiffen and total peripheral resistance increases. As a result, left ventricular afterload increases and so does systolic blood pressure (Avolio et al., 1983).

Advancing age is also associated with a premature timing of arterial wave reflection. In the circulatory system with arterial branching and tapering, a forward-traveling pressure wave generated from the left ventricle encounters the discontinuity of vascular impedance/resistance and reflects back a portion of the incident waves (Nichols et al., 2005). With the age-related increase in aortic pulse wave velocity, a reflected pressure wave collides prematurely with the incident pressure waves, thus leading to the augmentation of systolic and attenuation of diastolic pressures (Nichols et al., 2005). As discussed above, dynamic CA is effective in dampening changes in arterial pressure only at low frequency $(<0.1 \mathrm{~Hz})$ (Zhang et al., 1998). Therefore, age-related increase in hemodynamic pulsatility at cardiac frequency may be transmitted passively (i.e., without the counteraction of CA) into the brain and lead to brain structural damage.

Cerebrovascular impedance is elevated in the elderly while the brain is exposed to a greater magnitude of hemodynamic pulsatility (Zhu et al., 2010). Vascular impedance represents an opposition to pulsatile blood flow and is determined by the intrinsic property (i.e., elasticity) and diameter (i.e., resistance) of blood vessels (O'Rourke and Taylor, 1967). Age-related increases in cerebrovascular impedance with the concurrent elevations in CBF pulsatility observed in the basal cerebral arteries may reflect a compensatory mechanism of cerebral vasculature which attenuates the transmission of $\mathrm{CBF}$ pulsatility into the delicate microcirculation. In support of this, vascular adaptations to hemodynamic pulsatility have been shown from the peripheral vasculature (Laurent et al., 2009). For example, central arterial stiffness and higher systolic blood pressure were positively associated with thicker wall of resistance vessels relative to the lumen diameter (Muiesan et al., 2013). Moreover, greater central pulse pressure was positively associated with wall-to-lumen ratio of retinal arterioles which is an independent risk factor for stroke (Wong et al., 2001; Ott et al., 2013). Such adaptations of the microvasculature increase vascular impedance which would attenuate the transmission of hemodynamic pulsatility, reduce vascular damage, and facilitate oxygen extraction. However, these adaptations may occur at the expense of increases in vascular resistance and thus reduction in steady-state brain perfusion. Indeed, central arterial stiffness is negatively correlated with CBF in the deep white matter where a high prevalence of $\mathrm{WMH}$ are observed (Tarumi et al., 2011). Moreover, central arterial stiffness and pressure pulsatility are associated with higher prevalence of subcortical infarct, atrophy of brain parenchyma, and greater levels of brain amyloid plaques (Mitchell et al., 2011; Hughes et al., 2013; Nation et al., 2013). These findings collectively indicate the potential importance of age-related changes in central hemodynamics to cerebrovascular remodeling and structural brain changes. In addition, prevention of central arterial aging may alleviate pulsatile-induced cerebral microvascular disease and age-related cognitive decline.

\section{AEROBIC EXERCISE AND THE BRAIN STRUCTURE AND FUNCTION}

There is an increasing recognition that habitual aerobic exercise enhances cognitive function and attenuates age-related deterioration of brain structure. Earlier studies in animals showed the greater benefits of voluntary aerobic exercise in improving cognitive function when compared with other stimuli such as expanded learning opportunities (van Praag et al., 1999). Furthermore, exercise-related improvements in cognitive function were associated with the neurogenesis of hippocampal dentate gyrus in the adult mouse (van Praag et al., 1999). Human studies also demonstrated that regular walking increased the hippocampal size and improved the memory performance in the previously sedentary elderly individuals (Erickson et al., 2011).

Regular aerobic exercise preserves the structural integrity of white matter (Gons et al., 2013; Tseng et al., 2013). As assessed by diffusion tensor MR imaging, Master's athletes, who have participated in a lifelong high intensity, high volume aerobic exercise training, attenuated age-related reductions in axonal fiber integrity, as shown by higher fractional anisotropy and lower mean diffusivity in the network of front-to-back connections (Tseng et al., 2013). Moreover, there was a strong positive correlation between maximal oxygen uptake and fractional anisotropy in the left superior longitudinal fasciculus (Tseng et al., 2013). These findings suggest that regular aerobic exercise preserves the microstructural integrity of white matter that is responsible for 
visuospatial function, motor control, and coordination (Tseng et al., 2013).

Exercise-related improvements in brain function and structure may be conferred by the concurrent adaptations in vascular function and structure. Aerobic exercise increases the peripheral levels of growth factors (e.g., BDNF, IFG-1, and VEGF) which cross the blood-brain barrier (BBB) and stimulate neurogenesis and angiogenesis (Trejo et al., 2001; Lee et al., 2002; Fabel et al., 2003; Lopez-Lopez et al., 2004). Consistent with this, exercise-related enlargement of hippocampus was accompanied by increases in cerebral blood volume and capillary densities (Pereira et al., 2007). Enhanced cerebral perfusion may not only facilitate the delivery of energy substrates, but also lower the risk of vascularrelated brain damages, including WMH and silent infarct (Tseng et al., 2013). Furthermore, regular aerobic exercise is associated with lower levels of $A \beta$ deposition in individuals with APOE4 positive (Head et al., 2012), which may also reduce the risk of cerebral amyloid angiopathy and microbleeds (Poels et al., 2010).

Regular aerobic exercise ameliorates endothelial dysfunction and central arterial stiffness (Seals et al., 2008). As discussed above, central arteries serve as an important interface between the cerebral and peripheral circulations (Nichols et al., 2005). A recent study which compared middle-aged endurance-trained and sedentary adults demonstrated that higher aerobic fitness in endurance-trained adults was correlated positively with better cognitive performance and negatively with central arterial stiffness, independent of other lifestyle factors (e.g., sleep and diet) (Tarumi et al., 2013). Furthermore, lower central arterial stiffness, as assessed by carotid artery distensibility, showed positive associations with cognitive performance and resting CBF in the occipito-parietal area (Tarumi et al., 2013). In addition, exercise-related enhancement of endothelial function may facilitate neurovascular coupling and protect cerebral arteries from atherogenesis. In humans, endothelium-dependent vasodilatation measured from the peripheral (e.g., brachial and radial) arteries is considered a systemic index of vascular endothelial function (Celermajer et al., 1994; DeSouza et al., 2000). If this is also the case for cerebral circulation, exercise-related improvement in endothelial function is likely to benefit CBF regulation, structural integrity of BBB, and cognitive outcome. Currently, there is a relative lack of data as to the impact of exercise-related improvement in endothelial function on brain structure and function. Future studies are needed to confirm whether a reversal of endothelial dysfunction translates into better brain structural and cognitive outcomes.

\section{SUMMARY}

$\mathrm{AD}$ and cerebrovascular disease often coexist in the aging brain. Traditional view that $\mathrm{AD}$ and $\mathrm{VaD}$ are two divergent clinical entities with few or no overlap between their pathologies is challenged by an accumulating body of evidence indicating a close association between vascular disease and the risk of AD development. More recently, physiological studies also have demonstrated that vascular dysregulation of $\mathrm{CBF}$ elevate the risk of both cerebrovascular disease and AD. Specifically, presence of central arterial stiffness and endothelial dysfunction elevates the risk of pressure pulsatility or flow-related damage to brain structure and function. Further study of these modifiable risk factors through pharmacological or non-pharmacological approaches is likely to be important for developing an effective prevention or treatment for AD. In particular, further mechanistic study of the effect of regular aerobic exercise on arterial aging, brain perfusion and structure may provide new insights into how to prevent or slow the age-related cognitive decline, $\mathrm{AD}$, and other age-related cerebrovascular diseases.

\section{AUTHOR CONTRIBUTIONS}

Takashi Tarumi drafted the manuscript. Rong Zhang revised the manuscript critically for important intellectual content. Rong Zhang and Takashi Tarumi approved the final version of the manuscript.

\section{ACKNOWLEDGMENTS}

This study was supported in part by the NIH grant R01AG033106-01and R01HL102457.

\section{REFERENCES}

Attwell, D., Buchan, A. M., Charpak, S., Lauritzen, M., Macvicar, B. A., and Newman, E. A. (2010). Glial and neuronal control of brain blood flow. Nature 468, 232-243. doi: 10.1038/nature09613

Avolio, A. P., Chen, S. G., Wang, R. P., Zhang, C. L., Li, M. F., and O'Rourke, M. F. (1983). Effects of aging on changing arterial compliance and left ventricular load in a northern Chinese urban community. Circulation 68, 50-58. doi: 10.1161/01.CIR.68.1.50

Breteler, M. M. (2000). Vascular risk factors for Alzheimer's disease: an epidemiologic perspective. Neurobiol. Aging 21, 153-160. doi: 10.1016/S01974580(99)00110-4

Bu, G. (2009). Apolipoprotein E and its receptors in Alzheimer's disease: pathways, pathogenesis and therapy. Nat. Rev. Neurosci. 10, 333-344. doi: $10.1038 / \mathrm{nrn} 2620$

Casserly, I., and Topol, E. (2004). Convergence of atherosclerosis and Alzheimer's disease: inflammation, cholesterol, and misfolded proteins. Lancet 363, 1139-1146. doi: 10.1016/S0140-6736(04)15900-X

Celermajer, D. S., Sorensen, K. E., Bull, C., Robinson, J., and Deanfield, J. E. (1994). Endothelium-dependent dilation in the systemic arteries of asymptomatic subjects relates to coronary risk factors and their interaction. J. Am. Coll. Cardiol. 24, 1468-1474. doi: 10.1016/0735-1097(94)90141-4

Christie, R., Yamada, M., Moskowitz, M., and Hyman, B. (2001). Structural and functional disruption of vascular smooth muscle cells in a transgenic mouse model of amyloid angiopathy. Am. J. Pathol. 158, 1065-1071. doi: 10.1016/S0002-9440(10)64053-9

Claassen, J. A., Colier, W. N., and Jansen, R. W. (2006). Reproducibility of cerebral blood volume measurements by near infrared spectroscopy in 16 healthy elderly subjects. Physiol. Meas. 27, 255-264. doi: 10.1088/0967-3334/27/3/004

Claassen, J. A., Diaz-Arrastia, R., Martin-Cook, K., Levine, B. D., and Zhang, R. (2009). Altered cerebral hemodynamics in early Alzheimer disease: a pilot study using transcranial Doppler. J. Alzheimers Dis. 17, 621-629. doi: 10.3233/JAD2009-1079

Davignon, J., and Ganz, P. (2004). Role of endothelial dysfunction in atherosclerosis. Circulation 109, III27-III32. doi: 10.1161/01.CIR.0000131515.03336.f8

Dede, D. S., Yavuz, B., Yavuz, B. B., Cankurtaran, M., Halil, M., Ulger, Z., et al. (2007). Assessment of endothelial function in Alzheimer's disease: is Alzheimer's disease a vascular disease? J. Am. Geriatr. Soc. 55, 1613-1617. doi: 10.1111/j.1532-5415.2007.01378.x

de la Torre, J. C. (2004). Is Alzheimer's disease a neurodegenerative or a vascular disorder? Data, dogma, and dialectics. Lancet Neurol. 3, 184-190. doi: 10.1016/S1474-4422(04)00683-0

DeSouza, C. A., Shapiro, L. F., Clevenger, C. M., Dinenno, F. A., Monahan, K. D., Seals, D.R., et al. (2000). Regular aerobic exercise prevents and restores age-related declines in endothelium-dependent vasodilation in healthy men. Circulation 102, 1351-1357. doi: 10.1161/01.CIR.102.12.1351 
Dietrich, H. H., Kajita, Y., and Dacey, R. G. Jr. (1996). Local and conducted vasomotor responses in isolated rat cerebral arterioles. Am J Physiol. 271, H1109-H1116.

Erickson, K. I., Voss, M. W., Prakash, R. S., Basak, C., Szabo, A., Chaddock, L., et al. (2011). Exercise training increases size of hippocampus and improves memory. Proc. Natl. Acad. Sci. U.S.A. 108, 3017-3022. doi: 10.1073/pnas.1015950108

Fabel, K., Tam, B., Kaufer, D., Baiker, A., Simmons, N., Kuo, C. J., et al. (2003). VEGF is necessary for exercise-induced adult hippocampal neurogenesis. Eur. J. Neurosci. 18, 2803-2812. doi: 10.1111/j.1460-9568.2003.03041.x

Faraci, F. M., and Heistad, D. D. (1990). Regulation of large cerebral arteries and cerebral microvascular pressure. Circ. Res. 66, 8-17. doi: 10.1161/01.RES.66.1.8

Fujii, K., Heistad, D. D., and Faraci, F. M. (1991). Flow-mediated dilatation of the basilar artery in vivo. Circ. Res. 69, 697-705. doi: 10.1161/01.RES.69.3.697

Gons, R. A., Tuladhar, A. M., de Laat, K. F., van Norden, A. G., van Dijk, E. J., Norris, D. G., et al. (2013). Physical activity is related to the structural integrity of cerebral white matter. Neurology 81, 971-976. doi: 10.1212/WNL.0b013e3182a43e33

Gorelick, P. B., Scuteri, A., Black, S. E., Decarli, C., Greenberg, S. M., Iadecola, C., et al. (2011). Vascular contributions to cognitive impairment and dementia: a statement for healthcare professionals from the american heart association/american stroke association. Stroke 42, 2672-2713. doi: 10.1161/STR.0b013e3182299496

Hardy, J., and Selkoe, D. J. (2002). The amyloid hypothesis of Alzheimer's disease: progress and problems on the road to therapeutics. Science 297, 353-356. doi: $10.1126 /$ science. 1072994

Head, D., Bugg, J. M., Goate, A. M., Fagan, A. M., Mintun, M. A., Benzinger, T., et al. (2012). Exercise engagement as a moderator of the effects of APOE genotype on amyloid deposition. Arch. Neurol. 69, 636-643. doi: 10.1001/archneurol.2011.845

Hossmann, K. A. (1994). Viability thresholds and the penumbra of focal ischemia. Ann. Neurol. 36, 557-565. doi: 10.1002/ana.410360404

Hughes, T. M., Kuller, L. H., Barinas-Mitchell, E. J., Mackey, R. H., McDade, E. M., Klunk, W. E., et al. (2013). Pulse wave velocity is associated with beta-amyloid deposition in the brains of very elderly adults. Neurology 81, 1711-1718. doi: 10.1212/01.wnl.0000435301.64776.37

Kim, J., Basak, J. M., and Holtzman, D. M. (2009). The role of apolipoprotein E in Alzheimer's disease. Neuron 63, 287-303. doi: 10.1016/j.neuron.2009.06.026

Kivipelto, M., Helkala, E. L., Laakso, M. P., Hanninen, T., Hallikainen, M., Alhainen, K., et al. (2001). Midlife vascular risk factors and Alzheimer's disease in later life: longitudinal, population based study. BMJ 322, 1447-1451. doi: 10.1136/bmj.322.7300.1447

Lassen, N. A. (1959). Cerebral blood flow and oxygen consumption in man. Physiol. Rev. 39, 183-238.

Laurent, S., Briet, M., and Boutouyrie, P. (2009). Large and small artery crosstalk and recent morbidity-mortality trials in hypertension. Hypertension 54, 388-392. doi: 10.1161/HYPERTENSIONAHA.109.133116

Lautenschlager, N. T., Cox, K. L., Flicker, L., Foster, J. K., van Bockxmeer, F. M., Xiao, J., et al. (2008). Effect of physical activity on cognitive function in older adults at risk for Alzheimer disease: a randomized trial. JAMA 300, 1027-1037. doi: 10.1001/jama.300.9.1027

Lee, J., Duan, W., and Mattson, M. P. (2002). Evidence that brainderived neurotrophic factor is required for basal neurogenesis and mediates, in part, the enhancement of neurogenesis by dietary restriction in the hippocampus of adult mice. J. Neurochem. 82, 1367-1375. doi: 10.1046/j.1471-4159.2002.01085.x

Leenders, K. L., Perani, D., Lammertsma, A. A., Heather, J. D., Buckingham, P., Healy, M. J., et al. (1990). Cerebral blood flow, blood volume and oxygen utilization. Normal values and effect of age. Brain 113(Pt 1), 27-47. doi: 10.1093/brain/113.1.27

Liu, J., Zhu, Y. S., Khan, M. A., Brunk, E., Martin-Cook, K., Weiner, M. F., et al. (2013). Global brain hypoperfusion and oxygenation in amnestic mild cognitive impairment. Alzheimers Dement. doi: 10.1016/j.jalz.2013.04.507. [Epub ahead of print].

Lopez-Lopez, C., LeRoith, D., and Torres-Aleman, I. (2004). Insulin-like growth factor I is required for vessel remodeling in the adult brain. Proc. Natl. Acad. Sci. U.S.A. 101, 9833-9838. doi: 10.1073/pnas.0400337101

Madsen, P. L., and Secher, N. H. (1999). Near-infrared oximetry of the brain. Prog. Neurobiol. 58, 541-560. doi: 10.1016/S0301-0082(98)00093-8
Mahley, R. W., and Rall, S. C. Jr. (2000). Apolipoprotein E: far more than a lipid transport protein. Annu. Rev. Genomics Hum. Genet. 1, 507-537. doi: 10.1146/annurev.genom.1.1.507

Matsuda, H. (2001). Cerebral blood flow and metabolic abnormalities in Alzheimer's disease. Ann. Nucl. Med. 15, 85-92. doi: 10.1007/BF02988596

Matsushita, K., Kuriyama, Y., Nagatsuka, K., Nakamura, M., Sawada, T., and Omae, T. (1994). Periventricular white matter lucency and cerebral blood flow autoregulation in hypertensive patients. Hypertension 23, 565-568. doi: 10.1161/01.HYP.23.5.565

Mitchell, G. F., van Buchem, M. A., Sigurdsson, S., Gotal, J. D., Jonsdottir, M. K., Kjartansson, O., et al. (2011). Arterial stiffness, pressure and flow pulsatility and brain structure and function: the Age, Gene/Environment SusceptibilityReykjavik study. Brain 134, 3398-3407. doi: 10.1093/brain/awr253

Muiesan, M. L., Salvetti, M., Rizzoni, D., Paini, A., Agabiti-Rosei, C., Aggiusti, C., et al. (2013). Pulsatile hemodynamics and microcirculation: evidence for a close relationship in hypertensive patients. Hypertension 61, 130-136. doi: 10.1161/HYPERTENSIONAHA.111.00006

Murkin, J. M., and Arango, M. (2009). Near-infrared spectroscopy as an index of brain and tissue oxygenation. Br. J. Anaesth. 103(Suppl. 1), i3-i13. doi: 10.1093/bja/aep299

Nation, D. A., Edland, S. D., Bondi, M. W., Salmon, D. P., Delano-Wood, L., Peskind, E. R., et al. (2013). Pulse pressure is associated with Alzheimer biomarkers in cognitively normal older adults. Neurology 81, 2024-2027. doi: 10.1212/01.wnl.0000436935.47657.78

Nichols, W. W., O’Rourke, M., and Vlachopoulos, C. (2005). “McDonald's Blood Flow in Arteries". London: Hodder Arnold.

Niwa, K., Kazama, K., Younkin, L., Younkin, S. G., Carlson, G. A., and Iadecola, C. (2002). Cerebrovascular autoregulation is profoundly impaired in mice overexpressing amyloid precursor protein. Am. J. Physiol. Heart Circ. Physiol. 283, H315-H323. doi: 10.1152/ajpheart.00022.2002

O’Rourke, M. F., and Taylor, M. G. (1967). Input impedance of the systemic circulation. Circ Res. 20, 365-380. doi: 10.1161/01.RES.20.4.365

Okamoto, Y., Yamamoto, T., Kalaria, R. N., Senzaki, H., Maki, T., Hase, Y., et al. (2012). Cerebral hypoperfusion accelerates cerebral amyloid angiopathy and promotes cortical microinfarcts. Acta Neuropathol. 123, 381-394. doi: 10.1007/s00401-011-0925-9

Ornish, D., Brown, S. E., Scherwitz, L. W., Billings, J. H., Armstrong, W. T., Ports, T. A., et al. (1990). Can lifestyle changes reverse coronary heart disease? The lifestyle heart trial. Lancet 336, 129-133. doi: 10.1016/0140-6736(90)91656-U

Ott, C., Raff, U., Harazny, J. M., Michelson, G., and Schmieder, R. E. (2013). Central pulse pressure is an independent determinant of vascular remodeling in the retinal circulation. Hypertension 61, 1340-1345. doi: 10.1161/HYPERTENSIONAHA.111.00617

Paulson, O. B., Strandgaard, S., and Edvinsson, L. (1990). Cerebral autoregulation. Cerebrovasc. Brain Metab. Rev. 2, 161-192.

Pereira, A. C., Huddleston, D. E., Brickman, A. M., Sosunov, A. A., Hen, R., McKhann, G. M., et al. (2007). An in vivo correlate of exercise-induced neurogenesis in the adult dentate gyrus. Proc. Natl. Acad. Sci. U.S.A. 104, 5638-5643. doi: 10.1073/pnas.0611721104

Poels, M. M., Vernooij, M. W., Ikram, M. A., Hofman, A., Krestin, G. P., van der Lugt, A., et al. (2010). Prevalence and risk factors of cerebral microbleeds: an update of the Rotterdam scan study. Stroke 41, S103-S106. doi: 10.1161/STROKEAHA.110.595181

Querfurth, H. W., and LaFerla, F. M. (2010). Alzheimer's disease. N. Engl. J. Med. 362, 329-344. doi: 10.1056/NEJMra0909142

Roher, A. E., Esh, C., Kokjohn, T. A., Kalback, W., Luehrs, D. C., Seward, J. D., et al. (2003). Circle of willis atherosclerosis is a risk factor for sporadic Alzheimer's disease. Arterioscler. Thromb. Vasc. Biol. 23, 2055-2062. doi: 10.1161/01.ATV.0000095973.42032.44

Rowbotham, G. F., and Little, E. (1965). Circulations of the cerebral hemispheres. Br. J. Surg. 52, 8-21. doi: 10.1002/bjs.1800520104

Rowley, A. B., Payne, S. J., Tachtsidis, I., Ebden, M. J., Whiteley, J. P., Gavaghan, D. J., et al. (2007). Synchronization between arterial blood pressure and cerebral oxyhaemoglobin concentration investigated by wavelet cross-correlation. Physiol. Meas. 28, 161-173. doi: 10.1088/0967-3334/28/2/005

Seals, D. R., Desouza, C. A., Donato, A. J., and Tanaka, H. (2008). Habitual exercise and arterial aging. J. Appl. Physiol. (1985) 105, 1323-1332. doi: 10.1152/japplphysiol.90553.2008 
Selkoe, D. J. (1991). The molecular pathology of Alzheimer's disease. Neuron 6, 487-498. doi: 10.1016/0896-6273(91)90052-2

Selkoe, D. J. (2012). Preventing Alzheimer's disease. Science 337, 1488-1492. doi: $10.1126 /$ science. 1228541

Snowdon, D. A., Greiner, L. H., Mortimer, J. A., Riley, K. P., Greiner, P. A., and Markesbery, W. R. (1997). Brain infarction and the clinical expression of Alzheimer disease. The nun study. JAMA 277, 813-817. doi: 10.1001/jama.1997.03540340047031

Tarumi, T., Gonzales, M. M., Fallow, B., Nualnim, N., Pyron, M., Tanaka, H., et al. (2013). Central artery stiffness, neuropsychological function, and cerebral perfusion in sedentary and endurance-trained middle-aged adults. J. Hypertens. 31, 2400-2409. doi: 10.1097/HJH.0b013e328364decc

Tarumi, T., Shah, F., Tanaka, H., and Haley, A. P. (2011). Association between central elastic artery stiffness and cerebral perfusion in deep subcortical gray and white matter. Am. J. Hypertens. 24, 1108-1113. doi: 10.1038/ajh.2011.101

Thies, W., and Bleiler, L. (2013). 2013 Alzheimer's disease facts and figures. Alzheimers Dement. 9, 208-245. doi: 10.1016/j.jalz.2013.02.003

Thomas, T., Thomas, G., McLendon, C., Sutton, T., and Mullan, M. (1996). betaAmyloid-mediated vasoactivity and vascular endothelial damage. Nature 380, 168-171. doi: 10.1038/380168a0

Trejo, J. L., Carro, E., and Torres-Aleman, I. (2001). Circulating insulin-like growth factor I mediates exercise-induced increases in the number of new neurons in the adult hippocampus. J. Neurosci. 21, 1628-1634.

Tseng, B. Y., Gundapuneedi, T., Khan, M. A., Diaz-Arrastia, R., Levine, B. D., Lu, H., et al. (2013). White matter integrity in physically fit older adults. Neuroimage 82, 510-516. doi: 10.1016/j.neuroimage.2013.06.011

van Beek, A. H., Claassen, J. A., Rikkert, M. G., and Jansen, R. W. (2008). Cerebral autoregulation: an overview of current concepts and methodology with special focus on the elderly. J. Cereb. Blood Flow Metab. 28, 1071-1085. doi: $10.1038 /$ jcbfm. 2008.13

van Beek, A. H., Sijbesma, J. C., R. W. Jansen, Rikkert, M. G., and Claassen, J. A. (2010). Cortical oxygen supply during postural hypotension is further decreased in Alzheimer's disease, but unrelated to cholinesterase-inhibitor use. J. Alzheimers Dis. 21, 519-526. doi: 10.3233/JAD-2010-100288

van Praag, H., Kempermann, G., and Gage, F. H. (1999). Running increases cell proliferation and neurogenesis in the adult mouse dentate gyrus. Nat. Neurosci. 2, 266-270. doi: 10.1038/6368

Wilson, P. W., Myers, R. H., Larson, M. G., Ordovas, J. M., Wolf, P. A., and Schaefer, E. J. (1994). Apolipoprotein E alleles, dyslipidemia, and coronary heart disease. The Framingham Offspring Study. JAMA 272, 1666-1671. doi: 10.1001/jama.1994.03520210050031
Wilson, P. W., Schaefer, E. J., Larson, M. G., and Ordovas, J. M. (1996). Apolipoprotein E alleles and risk of coronary disease. A meta-analysis. Arterioscler. Thromb. Vasc. Biol. 16, 1250-1255. doi: 10.1161/01.ATV.16.10.1250

Wong, T. Y., Klein, R., Couper, D. J., Cooper, L. S., Shahar, E., Hubbard, L. D., et al. (2001). retinal microvascular abnormalities and incident stroke: the atherosclerosis risk in communities study. Lancet 358, 1134-1140. doi: 10.1016/S0140-6736(01)06253-5

Zazulia, A. R., Videen, T. O., Morris, J. C., and Powers, W. J. (2010). Autoregulation of cerebral blood flow to changes in arterial pressure in mild Alzheimer's disease. J. Cereb. Blood Flow Metab. 30, 1883-1889. doi: 10.1038/jcbfm.2010.135

Zhang, R., Zuckerman, J. H., Giller, C. A., and Levine, B. D. (1998). Transfer function analysis of dynamic cerebral autoregulation in humans. Am. J. Physiol. 274, $\mathrm{H} 233-\mathrm{H} 241$.

Zhang, R., Zuckerman, J. H., and Levine, B. D. (2000). Spontaneous fluctuations in cerebral blood flow: insights from extended-duration recordings in humans. Am. J. Physiol. Heart Circ. Physiol. 278, H1848-H1855.

Zhang, X., Zhou, K., Wang, R., Cui, J., Lipton, S. A., Liao, F. F., et al. (2007). Hypoxia-inducible factor lalpha (HIF-1alpha)-mediated hypoxia increases BACE1 expression and beta-amyloid generation. J. Biol. Chem. 282, 10873-10880. doi: 10.1074/jbc.M608856200

Zhu, Y. S., Tseng, B. Y., Shibata, S., Levine, B. D., and Zhang, R. (2010). Increases in cerebrovascular impedance in older adults. J. Appl. Physiol. (1985) 111, 376-381. doi: 10.1152/japplphysiol.01418.2010

Conflict of Interest Statement: The authors declare that the research was conducted in the absence of any commercial or financial relationships that could be construed as a potential conflict of interest.

Received: 30 November 2013; accepted: 05 January 2014; published online: 21 January 2014.

Citation: Tarumi T and Zhang $R$ (2014) Cerebral hemodynamics of the aging brain: risk of Alzheimer disease and benefit of aerobic exercise. Front. Physiol. 5:6. doi: 10.3389/fphys.2014.00006

This article was submitted to Integrative Physiology, a section of the journal Frontiers in Physiology.

Copyright (C) 2014 Tarumi and Zhang. This is an open-access article distributed under the terms of the Creative Commons Attribution License (CC BY). The use, distribution or reproduction in other forums is permitted, provided the original author(s) or licensor are credited and that the original publication in this journal is cited, in accordance with accepted academic practice. No use, distribution or reproduction is permitted which does not comply with these terms. 